\title{
Déterminants de virulence chez les bacilles à Gram négatif
}

Les bacilles à Gram négatif ont différents types d'interactions avec les tissus infectés, allant de l'adhésion simple à l'envahissement et à la lyse cellulaire. Ces interactions reflètent la synthèse par les microorganismes de divers types de substances actives et conditionnent la pathogénicité.

\section{Philippe Sansonetti Chargé de recherches à l'Institut Pasteur}

\section{ADRESSE}

P. Sansonetti : Service des entérobactéries, Inserm U. 199, Institut Pasteur, 28, rue du es bacilles à Gram négatif (BG-) sont une cause majeure d'infection chez l'homme. Ces infections sont d'une extraordinaire variété par la diversité des espèces bactériennes en cause, par leurs mécanismes physiopathologiques qui peuvent impliquer des microorganismes à multiplication extracellulaire ou au contraire intracellulaire, par leur topographie, leur gravité, la nature de l'hôte infecté. Cette variété reflète, pour l'essentiel, l'expression par la bactérie de facteurs de pathogénicité lui permettant de franchir les divers obstacles des défenses spécifiques et non spécifiques de l'organisme. Elle peut alors directement accéder aux tissus cibles ou y laisser diffuser des toxines qui vont y créer des altérations cellulaires temporaires ou définitives. Cette variété peut aussi refléter la faillite primitive ou secondaire des défenses immunitaires. Les infections systémiques à BG- sont en effet prédominantes au cours des agranulocytoses. Un modèle théorique prenant en compte l'ensemble des étapes possibles de l'infection, depuis la colonisation d'une surface muqueuse jusqu'à l'envahissement des viscères par dissémination hématogène, peut être bâti. Il permet un classement des BG- en différents " pathovars " c'est-à-dire en groupements de microorganis- mes qui, bien que taxonomiquement éloignés, présentent un pouvoir pathogène similaire. Inversement, une seule espèce telle Escherichia coli peut éclater en ces divers pathovars, prouvant à quel point son adaptabilité génétique lui a permis de coloniser diverses niches au sein de l'hôte humain. C'est d'ailleurs cette espèce qui sert de chef de file pour chacun des pathovars présentés dans le Tableau I.

I. Colonisation des muqueuses sans invasion tissulaire.

Muqueuses digestives. Dans certaines infections, la physiopathologie implique des phénomènes d'adhésion et de cytotonicité. Le modèle en est $E$. coli entérotoxinogène (ECET). Dans les régions à haut niveau d'hygiène, les ECET sont très rarement isolés. Ils sont par contre responsables d'un pourcentage élevé de diarrhées infantiles et de la diarrhée dite des voyageurs dans les régions intertropicales et dans certains pays en voie de développement. Il s'agit d'une diarrhée cholériforme dont la physiopathologie implique la colonisation de la surface muqueuse de l'intestin grêle par la bactérie à l'aide d'adhésines ou facteurs de colonisation (CF) puis la sécrétion d'exotoxines protéiques ou entérotoxines (LT et/ou ST). Il n'existe aucune lésion muqueuse. La/ou les toxi- 
nes agissent telles des hormones au niveau des entérocytes pour y déclencher une fuite hydroélectrolytique. Les ECET appartiennent à un nombre limité de sérotypes somatiques représentant sans doute un "réservoir " de souches en expansion clonale. La morphologie des adhésines est variable, qu'il s'agisse de filaments rigides appelés pili ou fimbriae qui sont des homopolymères constitués de la répétition d'une sous-unité protéique ou de structures plus fines, ondulantes. Ces adhésines permettent aux bactéries d'adhérer de façon spécifique à la bordure en brosse des entérocytes et de résister au processus d'élimination dû au péristaltisme intestinal. Les deux adhésines les plus fréquemment représentées à la surface des souches humaines sont appelées CFAI [1] et CFAII [2].

Les entérotoxines sont de deux types : thermolabile (LT) et thermostable (ST). La toxine LT, proche de la toxine cholérique, comprend deux sous-unités polypeptidiques. La sous-unité A (25 000 daltons) a la capacité de stimuler l'activité adénylcyclasique des cellules, en particulier entérocytaires. L'augmentation de l'AMPc intracellulaire agit de deux façons qui mènent à une fuite hydroélectrolytique dans la lumière intestinale [3]. La sousunité B (11 500 daltons) représentée par des aggrégats de cinq monomères est responsable de la spécificité d'adhésion de la toxine sur un ganglioside de la surface entérocytaire, le $\mathrm{GM}_{1}$. La toxine ST retrouvée dans les souches isolées chez l'homme est un petit polypeptide d'une vingtaine d'acides aminés qui entraîne une activation de la guanylate cyclase des entérocytes. Les gènes codant pour les toxines LT et ST ont jusqu'à présent été systématiquement retrouvés sur des plasmides $[4,5]$.

$V$. cholerae est un autre exemple de bactéries à Gram négatif agissant sur les muqueuses digestives par adhésion et cytotonicité. Les formes épidémique et pandémique du choléra sont dues à $V$. cholerae 01 qui possède deux biotypes, classique et El-Tor. La physiopathologie de l'infection qui peut conduire à une diarrhée hydroélectrolytique massive, voire mortelle, est en tout point superposable à celle causée par les ECET avec un système d'adhésion permettant la colonisation de la muqueuse de l'intestin grêle et une entérotoxine cytotonique, la toxine cholérique (CT).

Dans d'autres cas, la physiopathologie implique des phénomènes d'adhésion et de cytotoxicité. Un exemple en est $E$. coli entéropathogène (ECEP). Dans les régions

\begin{tabular}{|c|c|c|c|c|}
\hline \multicolumn{5}{|c|}{$\begin{array}{l}\text { DÉTERMINANTS DE VIRULENCE DES BACILLES GRAM NÉGATIF } \\
\text { GROUPEMENTS FONCTIONNELS DE PATHOGÉNICITÉ }\end{array}$} \\
\hline Pouvoir pathogène & Microorganisme & Maladie & Tissu atteint & Facteur(s) de pathogénicité \\
\hline Adhésion seule & $\begin{array}{l}\text { E. coli } \\
\text { uropathogènes }\end{array}$ & Cystite & Appareil urinaire & Adhésines \\
\hline $\begin{array}{l}\text { Adhésion et } \\
\text { cytotonicité }\end{array}$ & $\begin{array}{l}\text { E. coli } \\
\text { entérotoxinogènes } \\
\text { Vibrio cholerae }\end{array}$ & $\begin{array}{l}\text { "Turista " } \\
\text { Choléra }\end{array}$ & $\begin{array}{l}\text { Tube digestif } \\
\text { Tube digestif }\end{array}$ & $\begin{array}{l}\text { Adhésines + cytotonines } \\
\text { Adhésines + cytotonine }\end{array}$ \\
\hline \multirow{2}{*}{$\begin{array}{l}\text { Adhésion et } \\
\text { cytotoxicité }\end{array}$} & E. coli & Entérite aiguë & Tube digestif & Adhésine(s) + cytotoxine(s) \\
\hline & $\begin{array}{l}\text { E. coli } \\
\text { entérohémorragiques } \\
B \text {. pertussis } \\
P \text {. aeruginosa }\end{array}$ & $\begin{array}{l}\text { Diarrhée sanglante } \\
\text { Coqueluche } \\
\text { Colonisation }\end{array}$ & $\begin{array}{l}\text { Tube digestif } \\
\text { Trachée/bronches } \\
\text { Épithélium }\end{array}$ & $\begin{array}{l}\text { Adhésine }(s)+\text { cytotoxine }(s) \\
\text { Adhésines + cytotoxines } \\
\text { Adhésines + cytotoxines }\end{array}$ \\
\hline \multirow[t]{2}{*}{ Invasion tissulaire } & $\begin{array}{l}\text { Shigella } \\
\text { Salmonella }\end{array}$ & $\begin{array}{l}\text { Dysenterie bacillaire } \\
\text { Entérite aiguë, voire } \\
\text { fièvre typhoïde (S. } \\
\text { typhi) }\end{array}$ & $\begin{array}{l}\text { Tube digestif } \\
\text { Tube digestif }\end{array}$ & $\begin{array}{l}\text { Pénétration et multiplication } \\
\text { Pénétration intracellulaire }\end{array}$ \\
\hline & $\begin{array}{l}\text { Yersinia enterolitica } \\
\text { et } \\
\text { pseudotuberculosis }\end{array}$ & $\begin{array}{l}\text { Entérite ou adé- } \\
\text { nolymphite } \\
\text { mésentérique }\end{array}$ & Tube digestif & Pénétration intracellulaire \\
\hline \multirow[t]{2}{*}{$\begin{array}{l}\text { Suppuration, } \\
\text { dissémination } \\
\text { systémique, } \\
\text { localisation à } \\
\text { distance }\end{array}$} & E. coli & $\begin{array}{l}\text { Septicémies, } \\
\text { pyélonéphrites, } \\
\text { méningites }\end{array}$ & & $\begin{array}{l}\text { Capsule } \\
\text { LPS } \\
\text { Résistance au sérum } \\
\text { Captation du fer }\end{array}$ \\
\hline & $\begin{array}{l}\text { Autres } \\
\text { microorganismes }\end{array}$ & & & idem ? \\
\hline
\end{tabular}


industrialisées, les ECEP sont de plus en plus rarement isolés. Jadis responsables d'épidémies de gastroentérites chez les nourrissons, particulièrement en milieu hospitalier et dans les crèches, ils ont virtuellement disparu si l'on excepte quelques cas sporadiques. Ils représentent, par contre, une étiologie importante de diarrhée dans les régions tropicales et les pays en voie de développement. Là, ils donnent lieu à des gastroentérites aiguës ou à des diarrhées persistantes avec malabsorption secondaire. Ces souches font partie d'un nombre limité de sérotypes somatiques (055, 0111).

La physiopathologie de ce type d'infection est suggérée par des études anatomopathologiques effectuées au cours d'infections du nourrisson par des ECEP des sérotypes 0125 et $0119[6,7]$. La microscopie optique montre des microcolonies d'E. coli adhérant étroitement aux cellules du.sommet des villosités, avec effacement des cellules épithéliales. La microscopie électronique confirme l'adhésion étroite aux cellules épithéliales avec destruction locale de la bordure en brosse mais sans pénétration dans les cellules épithéliales. L'aspect des cellules épithéliales sous-jacentes aux microcolonies adhérentes est souvent très anormal. La capacité d'adhésion de ces souches est reflétée in vitro par une adhésion aux cellules Hep-2 en monocouche [8]. Il s'agit d'adhésines non fimbrillaires codées par des plasmides.

Les souches d'ECEP testées ne produisent ni toxine LT, ni ST. Des études préliminaires ayant montré que nombre de ces souches produisaient une cytotoxine détruisant les cellules Vero $(\mathrm{VT}+)[9]$, des travaux récents ont permis de préciser qu'il s'agissait d'une toxine apparentée à la toxine dysentérique (Shiga-like toxin 1 ou SLT1) qui est à la fois entérotoxique et cytotoxique et se présente donc comme un excellent candidat pour rendre compte de la diarrhée et des altérations épithéliales déjà décrites [10].
$E$. coli entérohémorragique (ECEH) est responsable d'un nouveau syndrome se présentant sous la forme d'une colite hémorragique, syndrome récemment décrit au cours d'intoxications alimentaires collectives aux USA et au Canada ou sous forme de cas sporadiques. Il a été rapporté à un sérogroupe inhabituel d' $E$. coli, 0157:H7 [11]. La présentation clinique la plus habituelle est celle d'une diarrhée suivie dans les 48 heures par l'émission de sang pur. L'observation endoscopique montre une muqueuse colique friable avec érythème, œdème, hémorragies et même ulcérations. Cet aspect, lié aux données radiologiques, évoque une colite ischémique aiguë. Ces souches produisent, pour la plupart, une quantité importante de cytotoxine récemment reconnue comme Shiga-like toxin 1 [16].

L'intérêt de ces souches productrices d'une cytotoxine s'est trouvé récemment renforcé par la démonstration d'une association entre la survenue du syndrome hémolytique et urémique, une des premières causes d'insuffisance rénale aiguë chez l'enfant, et la présence dans l'intestin d'E. coli produisant une activité cytotoxique sur les cellules Vero (VT +) [13]. Cette activité vérotoxique correspond à la toxine SLT1 [10]. Il s'agit le plus souvent de souches productrices du type ECEH (0157:H7) ou du type ECEP (026 en particulier). Cette situation établit un lien entre les syndromes hémolytiques et urémiques apparemment spontanés et ceux observés au décours des shigelloses, particulièrement après infection due à $S$. dysenteriae 1 qui produit de façon constitutive un haut niveau de toxine dysentérique (Shiga-toxin). Le rôle probable de cette famille de toxines dans la physiopathologie du syndrome hémolytique et urémique mérite une attention toute particulière. Muqueuse urinaire. En présence d'un arbre urinaire anatomiquement normal, sans obstacle, $E$. coli est responsable d'environ $90 \%$ des infections urinaires. Audelà de l'âge de trois mois, ces
Partin JL. A clinicopathoplogic study of enterocyte-adherent Escherichia coli : a cause of protracted diarrhea in infants. Gastroenterology $1982 ; 83: 441-54$. 
infections sont beaucoup plus fréquentes dans le sexe féminin. Le spectre de gravité clinique va de la colonisation asymptomatique à la cystite, puis à l'infection du haut appareil urinaire avec atteinte éventuelle du parenchyme rénal (pyélonéphrite). Certaines personnes présentant des infections récidivantes avec reflux vésico-urétéral développent une néphrite interstitielle chronique pouvant conduire à une insuffisance rénale chronique. Certains sérotypes d' $E$. coli sont significativement associés aux infections de l'arbre urinaire. Il existe d'évidentes différences entre les propriétés des $E$. coli isolés de pyélonéphrites, de cystites, voire de bactériémies asymptomatiques et des souches isolées de selles d'individus sains. Ces différences consistent essentiellement en la capacité des premières d'adhérer aux cellules uroépithéliales et de coloniser l'arbre urinaire. Cette propriété constante est bien corrélée avec la capacité de ces souches d'agglutiner des érythrocytes, en particulier humains, en présence de mannose (MRHA, mannose-resistant hemagglutination) [14]. Les adhésines de type MRHA les mieux caractérisées sont de "type $\mathrm{P}$ ". Elles sont exprimées par environ $80 \%$ des souches d' $E$. coli uropathogènes. Elles agglutinent les érythrocytes humains, sauf ceux du groupe antigénique $P$; il s'agit de pili dont le récepteur cellulaire est un globotétraoside dont le digalactoside terminal est $\alpha$-gal(1-4)-ß-gal. Ces adhésines $P$ et les protéines nécessaires à leur expression sont codées par un opéron chromosomique appelé PAP [15]. D'autres adhésines ont été décrites, appelées type X [16]. Certains microorganismes peuvent aussi donner lieu à des infections urinaires primitives : Proteus, Klebsiella. Des adhésines ont également été mises en évidence mais leur caractérisation n'a pas atteint le niveau de celles des Escherichia coli uropathogènes.

Muqueuse respiratoire. La coqueluche est caractérisée par une colonisation trachéobronchique par le germe respon- sable Bordetella pertussis. Les lésions consistent en une altération des cellules ciliées conduisant à des lésions nécrotiques de l'épithélium sans invasion de la muqueuse. Comme pour les ECEP, la physiopathologie de l'infection permet de postuler pour un système d'adhésion du microorganisme à l'épithélium et pour la production d'une ou plusieurs cytotoxines responsables des altérations tissulaires. L'hémagglutinine filamenteuse (FHA) est une structure fimbrillaire probablement impliquée dans le processus d'adhésion. La toxine coquelucheuse ou pertussigène est responsable de l'essentiel de l'activité cytotoxique de $B$. pertussis. Elle bloque par ADP-ribosylation la sous-unité inhibitrice de l'adénylcyclase de la membrane de la cellule-cible (voir nouvelle $\mathrm{m} / \mathrm{s} n^{\circ} 10$, vol. 2, p. 583). La production non régulée d'AMPc par la cellulecible entraîne sa mort [17].

Pseudomonas aeruginosa est un microorganisme pathogène essentiellement opportuniste présentant une étonnante faculté de colonisation des surfaces, en particulier de l'épithélium respiratoire au cours de situations telles la trachéotomie, la chirurgie des voies aériennes supérieures, l'hospitalisation prolongée de sujets infectés, âgés, dénutris ou la mucoviscidose. Ce potentiel de colonisation est lié à l'expression de pili clairement impliqués dans l'attachement aux cellules de mammiferes. Homopolymères formés d'une seule sous-unité protéique de 16 à 18000 daltons, ils représentent un groupe antigéniquement hétérogène [18]. Un autre facteur particulièrement important dans la colonisation des muqueuses au cours des infections chroniques est la production d'un exopolysaccharide capsulaire qui explique l'aspect mucoïde des souches isolées en de telles situations. Il s'agit d'acide mannuronique et guluronique, liés en 1-4 [19]. Par ailleurs, les isolats cliniques de $P$. aeruginosa produisent de nombreuses substances potentiellement impliquées dans la survenue des lésions tissulaires secondaires à la colonisation ou à la résistance aux mécanismes de défense cellulaires et humoraux.

Ces divers facteurs ont été largement analysés dans une revue récente [20]. Parmi ces substances, on peut citer : lipopolyoside, exotoxine $A$, enzymes protéolytiques, leucocidine, substances hémolytiques, exoenzyme $\mathrm{S}$.

En conclusion, un certain nombre de BG - sont susceptibles de coloniser les muqueuses sans les envahir mais en y créant, à des degrés divers, des lésions tissulaires. Ces divers pathovars permettent de regrouper artificiellement des microorganismes ayant un organotropisme différent comme il est rappelé dans le Tableau $I$.

\section{Invasion des muqueuses après effraction cellulaire}

C'est essentiellement au sein des entérobactéries entéropathogènes que se retrouvent les espèces invasives, c'est-à-dire celles capables d'induire leur propre phagocytose par les cellules épithéliales intestinales qui ne sont pas des cellules phagocytaires professionnelles : Shigella et $E$. coli entéroinvasifs (ECEI), Salmonella, Yersinia enterocolitica et $Y$. pseudotuberculosis [21]. Les Shigella envahissent le côlon grâce à une phagocytose induite au niveau des cellules épithéliales. Le processus d'invasion de la muqueuse comporte plusieurs étapes essentielles : accès à la surface des cellules épithéliales, dislocation de la bordure en brosse et invagination de la membrane cytoplasmique apicale avec internalisation de la bactérie dans une vacuole intracytoplasmique [22]. La membrane de la vacuole de phagocytose est alors rapidement lysée, permettant une multiplication bactérienne intracellulaire explosive et entraînant la mort rapide de la cellule hôte [23]. Un tel processus permet l'invasion des cellules épithéliales adjacentes et réalise un foyer infectieux qui déclenche dans le tissu conjonctif sous-jacent une intense réaction inflammatoire avec abcès et ulcérations. Ces lésions forment le substrat des symptômes de la dysenterie bacil- 


\section{RÉFÉRENCES}

8. Cravioto A, Gross RJ, Scotland SM, Rowe B. An adhesive factor found in strains of Escherichia coli belonging to the traditional infantile enteropathogenic serotypes. Current Microbiology 1979 ; 3 : 95-9.

9. Konowalchuk J, Speirs JI, Stavric S. Vero response to a cytotoxin of Escherichia coli. Infect Immun 1977 ; 18 : 775-9.

10. O'Brien A, Laveck GD, Thomson MR, Formal SB. Production of Shigella dysenteriae type 1 -like cytotoxin by Escherichia coli. $J$ Infect Dis 1982 ; 146 : 763-9.

11. Riley LW, Remis RS, Helgerson SD, et al. Hemorrhagic colitis associated with a rare Escherichia coli serotype. N Engl J Med 1983 ; 308: 681-5.

12. O'Brien A, Lively TA, Chen ME, Rothman SW, Formal SB. Escherichia coli 0157:H7 strains associated with haemorrhagic colitis in the United States produce a Shigella dysenteriae 1 (Shiga)-like cytotoxin. Lancet 1983 ; i : 702.

13. Karmali MA, Petric M, Steele BT, Lin C. Sporadic cases of haemolytic-uraemic syndrome associated with faecal cytotoxin and cytotoxin-producing Escherichia coli in stools. Lancet 1983 ; i : 619-20.

14. Hagberg L, Jodal U, Korhonnen TK Lidin-Janson G, Lindberg U, Svanborg-Eden C. Adhesion, hemagglutination and virulence of Escherichia coli causing urinary tract infections. Infect Immun 1981; 31 : 564-70.

15. Hull RA, Gill RE, Hsu P, Minshew BH, Falkow S. Construction and expression of recombinant plasmids encoding type 1 or Dmannose resistant pili from a urinary tract infection Escherichia coli isolate. Infect Immun 1981 ; 33 : 933-8.

16. Labigne-Roussel AF, Lark L, Schoolnik G, Falkow S. Cloning and expression of an afumbrial adhesin (AFA) responsible for $P$ blood group-independant, mannose-resistant hemagglutination from a pyelonephritic $E$. coli strain. Infect Immun 1984, 46 : 251-9.

17. Katada T, Ui M. Direct modification of the membrane adenylate cyclase system by islet-activating protein. Proc Natl Acad Sci USA $1982 ; 79: 3129-33$.

18.Paranchych W, Sastry PA, Frost LS, Carpenter M, Armstrong GD, Watts TH. Biochemical studies on pili isolated from Pseudomonas aenuginosa strain PAO. Can J Microbiol 1979 ; $25: 1175-81$

19. Evans LR, Linker A. Production and characterization of the slime polysaccharide of Pseudomonas aenuginosa. J Bacteriol 1973 ; 116 : 915-24.

20. Lory S, Tai PC. In : Current Topics in Microbiology and Immunology, Vol. 118. Berlin- laire. Les bactériémies sont tout à fait exceptionnelles au cours de cette infection. Chez les Shigella comme chez les $E$. coli entéroinvasifs, les étapes successives d'interaction entre la bactérie et la cellule hôte eucaryote sont à déterminisme extrachromosomique. Les étapes suivantes de survie et multiplication au sein des tissus sont codées par des gènes chromosomiques [24]. Ces espèces représentent donc un modèle dans lequel la cellule épithéliale intestinale est utilisée comme site de réplication de la bactérie invasive, expliquant le syndrome dysentérique intense, le caractère superficiel des lésions et l'absence habituelle de bactériémie.

A l'opposé, des microorganismes tels les Salmonella et les Yersinia pénètrent dans les cellules épithéliales intestinales, principalement dans la région iléale. Elles s'y répliquent peu et les utilisent comme moyen de passage transépithélial avant d'être prises en compte par les cellules phagocytaires professionnelles, et de coloniser les ganglions mésentériques. Le syndrome dysentérique observé est toujours modéré, voire absent. Par contre, le potentiel d'infection de parenchymes à distance est plus net, surtout pour les salmonelloses au cours desquelles les bactériémies, partant des ganglions mésentériques colonisés, sont fréquentes [21]. Le problème majeur pour ces microorganismes est donc celui de la survie intracellulaire, en particulier dans les cellules phagocytaires.

Salmonella typhimurium survit et se multiplie lentement dans les macrophages tout en restant piégée au sein d'une vacuole de phagocytose intacte et soumise à l'activité du contenu lysosomial après fusion. On ne connaît pas clairement les facteurs bactériens responsables de cette résistance. Jusqu'à présent, seule l'expression d'un lipopolyoside de surface complet (LPS) semble conférer une résistance significative à la bactéricidie due aux enzymes lytiques des lysosomes [25]. Ce LPS qui compose l'essentiel de la membrane externe est composé d'une portion lipidique et d'un polysaccharide complexe.

Yersinia enterocolitica et $Y$. pseudotuberculosis peuvent elles aussi survivre et se multiplier au sein des macrophages à l'intérieur de vacuoles de phagocytose. Les mécanismes de cette survie sont encore mal compris [25].

\section{Suppuration, dissémination localisations secondaires}

En l'absence de défaillance profonde des défenses immunitaires spécifiques et/ou non spécifiques, un $\mathrm{BG}$ - qui a accédé à une surface muqueuse ne peut y développer une infection sévère, voire donner lieu à une dissémination systémique, que s'il est susceptible de croître in vivo, de résister à ces mêmes défenses et de créer des lésions parenchymateuses. Ces trois propriétés seront successivement envisagées en prenant Escherichia coli comme modèle. Les données éparses disponibles concernant d'autres BG - seront parallèlement envisagées.

Capacité de crô̂tre au sein des tissus. La capacité d'un microorganisme à se multiplier dans les conditions retrouvées au sein des tissus de l'hôte est un facteur essentiel au développement du processus infectieux. Le facteur actuellement le mieux défini est la disponibilité en fer ferrique $\left(\mathrm{Fe}^{+++}\right)$. Pour croître dans de bonnes conditions, une bactérie doit disposer d'environ $10^{-5} \mathrm{M} \mathrm{Fe}^{+++}$. Or, le fer de l'organisme est séquestré dans des compartiments intracellulaires (ferritine, hémosidérine, hémine) ou chélaté dans le sérum par une protéine présentant un fort coefficient d'affinité, la transferrine. En conséquence, le taux de $\mathrm{Fe}^{+++}$sérique libre dans le sérum est très bas, de l'ordre de $10^{-18}$ M, essentiellement en équilibre avec la transferrine. Cette condition est incompatible avec toute croissance bactérienne au moins extracellulaire.

Nombreux sont les BG - testés qui produisent des sidérophores, molécules capables de fixer le $\mathrm{Fe}^{+++}$avec un coefficient d'affinité équivalent à celui de la trans- 
ferrine. Le complexe ainsi formé est repris par la bactérie au sein de laquelle le fer est libéré. Ces chélateurs de petit poids moléculaire varient dans leur structure. Il peut s'agir de phénolate, c'està-dire d'un trimère cyclique de 2 , 3-dihydroxybenzoillsérine, ou d'hydroxamate, petite molécule résultant de la condensation d'un citrate et de deux lysines hydroxylaminées. La biosynthèse de ces sidérophores est habituellement induite lorsque la concentration en $\mathrm{Fe}^{+++} \mathrm{du}$ milieu tombe audessous de $10 \mu \mathrm{M}$. L'entérochéline produite par $E$. coli et de nombreuses autres entérobactéries telles $S$. typhimurium et $K$. pneumoniae [26] est une molécule de type phénolate au même titre que les pyochélines produites par $P$. aeruginosa [27]. L'aérobactine, sidérophore de type hydroxamate, est fréquemment produite par des microorganismes pathogènes : souches d' $E$. coli isolées de pyélonéphrites ou de septicémie, isolats d'Enterobacter cloacae [26], souches hautement virulentes de $K$. pneumoniae (X. Nassif et P.J. Sansonetti, sous presse). Les gènes codant pour les enzymes nécessaires à la biosynthèse de l'aérobactine sont souvent situés sur des plasmides, contrairement à ceux codant pour les enzymes de la biosynthèse de l'entérocholine qui sont chromosomiques.

Capacité d'échapper aux défenses de l'hôte. Deux attributs essentiels rendent compte de la capacité des BG - à résister aux défenses de l'hôte : la résistance à l'activité bactéricide du sérum et la résistance à la phagocytose. La bactéricidie des BG - par le sérum est essentiellement due au complément, que la réaction soit médiée par les anticorps via la voie classique ou implique son action directe via la voie alterne. La membrane externe constitue la barrière à l'effet du complément. Le LPS en est l'élément majeur. On ne sait pas clairement comment le LPS protège la bactérie. Il est probable que le complexe d'attaque formé par le complément ne peut accéder à sa cible du fait de l'obstacle physique. De plus, certains plasmides tels ColV codant pour une protéine de membrane externe appelée Iss, ou R6-5 codant pour TraT, une autre protéine de membrane externe, jouent un rôle chez certaines souches pathogènes : $E$. coli, Salmonella, Shigella $[28,29]$. La phagocytose des BG - par les cellules phagocytaires professionnelles est efficacement prévenue lorsque le microorganisme produit une capsule. Celle-ci charge négativement la surface bactérienne, gênant ainsi l'interaction avec la surface des cellules eucaryotes. Elle gêne par ailleurs l'interaction des opsonines adsorbées à la surface bactérienne avec leurs récepteurs spécifiques sur la surface de la cellule phagocytaire. Chez les BG - , l'exemple typique est la capsule $\mathrm{K} \mathrm{d}^{\prime} E$. coli. Le sérogroupe K1 le plus souvent représenté est un polysaccharide acide composé d'acide $\mathrm{N}$-acétylneuraminique. $80 \%$ des souches d' $E$. coli isolées de méningites néonatales expriment le sérogroupe capsulaire $\mathrm{K} 1$ [30] ainsi que $40 \%$ des sou- ches isolées de septicémies [31]. Les souches restantes sont aussi capsulées mais de sérogroupes différents. La production d'une capsule antiphagocytaire est aussi un facteur essentiel de la virulence d'autres BG - à potentiel de multiplication extracellulaire et de dissémination septicémique : Klebsiella pneumoniae, Hemophilus influenzae, Pseudomonas aeruginosa.

Capacité de lyser les tissus de l'hôte. De nombreux facteurs d'origine bactérienne sont susceptibles de léser les tissus infectés y compris indirectement par le déclenchement de réactions inflammatoires ou de phénomènes immunopathologiques. Deux d'entre-eux méritent d'être individualisés :

(a) les hémolysines. Les hémolysines $\alpha$ sont les plus fréquentes chez $E$. coli. Elles représentent une famille de protéines acides, immunologiquement apparentées et nécessitant $\mathrm{Ca}^{++}$et $\mathrm{pH}$ neutre pour une activité optimale. Il existe plusieurs arguments à
Tableau II

\section{EFFETS BIOLOGIQUES DE L'ENDOTOXINE}

Effet pyrogène : pyrogènes endogènes libérés par les polynucléaires

Phénomène de Schwartzmann : local et général

Action sur des médiateurs humoraux :

- Coagulation (facteur de Hageman)

- Complément

- Kinine et dérivés

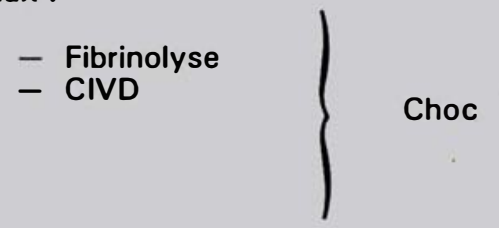

Action sur des systèmes cellulaires :

- Activation des plaquettes : thrombopénie

- Action sur les polynucléaires : neutropénie puis polynucléose

- Action sur monocytes, macrophages, cellules endothéliales, mastocytes et basophiles

Autres : hyperglycémie puis hypoglycémie ; induction d'interféron, de prostaglandines. 


\section{RÉFÉRENCES}

21. Formal SB, Hale TL, Sansonetti PJ. Invasive enteric pathogens. Rev Infed Dis 1983 ; 5 : S702-7.

22. Takeuchi A, Sprinz H, Labrec EH, et al. Experimental bacillary dysentery : an electron microscopic study of the response of the intestinal mucosa to bacterial invasion. $A m J$ Pathol $1965 ; 47: 1011-44$

23. Sansonetti PJ, Ryter A, Clerc P, Maurelli AT, Mounier J. Multiplication of Shigelle flexneri, within HeLa cells : Lysis of the phagocytic vacuole and plasmid-mediated contact hemolysis. Infect Immun 1986 ; 51 : 461-9.

24. Sansonetti PJ, Hale TL, Dammin GJ, Kapfer C, Collins HH, Formal SB. Alterations in the pathogenicity of Escherichia coli K 12 after transfer of plasmid and chromosomal genes from Shigella flerneri. Infect Immun 1983 ; 39 : 1392-402.

25. Brubaker RR. Mechanisms of bacterial virulence. Annu Rev Microbiol 1985 ; 39 : 21-50.

26. Griffiths E. Candidate virulence markers In : Susman S, ed. The Virulence of Escherichio coli. Society for General Microbiology. London : Academic Press, 1985 : 193-226.

27. Liu PV, Shokrani F. Biological activities of pyochelins : Iron chelating agents of Pseudomonas a aruginosa. Infect Immun 1978; 22 : 878-90.

28. Moll A, Manning PA, Timmis KN. Plasmid-determined resistance to serum bactericidal activity : a major outer membrane protein, the traT gene product, is responsible for plasmid-specified serum resistance in Escherichia coli. Infect Immun $1980 ; 28$ : 359-67.

29. Nilius AM, Savage DC. Serum resistance encoded by colicin V plasmids in Escherichia coli and its relationship to the plasmid transfer system. Infect Immun $1984 ; 43$ : 947-53.

30. Robbins JB, McCracken GH Jr, Gotschlich EC, Orskov F, Orskov I, Hanson LA. Escherichia coli K1 capsular polysaccharide associated with neonatal meningitis. $N$ Engl J Med 1974 ; 290 : 1216-20.

31. McCracken GH, Sarff LD, Globe MP, et al. Relation between Escherichia coli $\mathrm{K} 1$ capsular polysaccharide antigen and clinical outcome in neonatal meningitis. Lancet 1974; ii : 246-50.

32. Welch RA, Dellinger EP, Minshew B, Falkow S. Haemolysin contributes to virulence of extra-intestinal $E$. coli infections. Nature $1981 ; 294$ : 665-7.

33. McCabe WR. Endotoxin : microbiological, chemical, pathophysiologic and clinical correlations. In : Weinstein L, Field BN, ed. Saminars in Infectious Diseases (vol. III). New York : l'appui du rôle des hémolysines $\alpha$ dans la pathogénicité. Les souches isolées d'infections sont significativement plus fréquemment hémolytiques que les souches témoins. Les souches hémolytiques sont plus virulentes pour la souris et le rat que les souches non hémolytiques. Enfin et surtout, le transfert dans un isolat fécal d' $E$. coli d'un plasmide recombinant dans lequel ont été clonés les gènes codant pour l'hémolysine, augmente significativement la virulence de la souche réceptrice chez le rat [32]. Le mode d'action de ce type d'hémolysine n'est pas parfaitement élucidé tant au niveau moléculaire que physiopathologique. Elles agiraient directement par altération des membranes biologiques, soit sur les cellules parenchymateuses, soit sur les cellules phagocytaires (leucocidines). Elles pourraient aussi agir indirectement comme mécanisme additionnel de captation du fer par lyse érythrocytaire et libération du fer hémique. De nombreux autres BG - sont régulièrement hémolytiques : $V$. cholerae, $V$. parahemolyticus, B. pertussis.

(b) le lipopolyoside. Tous les BG - ont un LPS dont la portion lipidique, le lipide $\mathrm{A}$, est responsable de la toxicité et l'autre, polysaccharidique, supporte la spécificité somatique du microorganisme. Son rôle dans la pathogénicité, en tant qu'endotoxine, est extrêmement complexe. Ses effets biologiques essentiels sont résumés sur le Tableau II, p. 73 [33]. Ils peuvent être locaux, contribuant à la gravité des lésions tissulaires ou généraux, tel le choc endotoxinique. Il est encore difficile de dire ce que la spécificité antigénique portée par les chaînes latérales du LPS (antigène somatique) apporte d'originalité à la virulence de telle ou telle espèce. Il est, par contre, certain que l'essentiel de la toxicité du LPS réside dans sa portion lipidique, le lipide $A$. Dès lors qu'un BG - , quel qu'il soit, envahit des tissus, il a donc le potentiel par son endotoxine de créer des lésions locales majeures, voire des complications générales sévères ou mortelles

\section{Summary}

Gram negative bacilli are involved in a large array of infections depending on the host's status as well as on the virulence attributes expressed by the infectious microorganism. An attempt is made at classifying these various microorganisms according to the pathophysiology of the infectious process. Three major pathovars are defined. In the first pathovar, microorganisms colonize the mucosal surface without invasion. Two subsets can be defined according to the type of damage that is caused to mucosal tissues. Unexpectedly, enteropathogenic $E$. coli fall in the same pathovar as $B$. pertussis and $P$. aeruginosa. In the second pathovar, microorganisms invade the mucosal surface by penetrating into epithelial cells. This may be either a way to replicate within the mucosa which is the major site of the infection or to gain access to other organs through systemic dissemination after initial translocation through the mucosal tissues. In the third pathovar, microorganisms have virulence attributes that allow survival within tissues which is followed by escape from host defenses and creation of tissue lesions. These microorganisms have a potential for systemic dissemination through bacteremia or septicemia with subsequent infection of other organs. It is striking that $E$. coli has succeeded in designing virulence traits that allow it to belong to each of these three pathovars.

\section{TIRÉS A PART}

P. Sansonetti : Service des entérobactéries, Inserm U. 199, Institut Pasteur, 28, rue du Docteur-Roux, 75724 Paris Cedex 15. 